
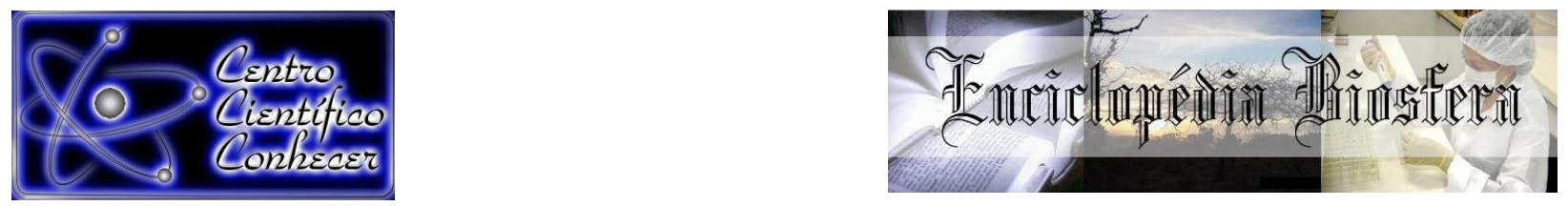

\title{
ESTIMAÇÃO DE VOLUMES DE Araucaria angustifolia (Bertol.) O. Kuntze POR FATORES DE FORMA EM CLASSES DIAMÉTRICAS E MODELOS DE REGRESSÃO
}

Carlos Roberto Sanquetta ${ }^{1}$; Marília Dolci ${ }^{2}$; Ana Paula Dalla Corte ${ }^{1}$; Mateus Niroh Inoue Sanquetta ${ }^{3}$; Allan Libanio Pelissari ${ }^{2}$

1 - Eng. Florestal, Dr., Professor da Universidade Federal do Paraná

2 - Eng. Florestal (marilia.dolci@gmail.com)

3 - Acadêmico de Engenharia Florestal da Universidade Federal do Paraná

Av. Lothário Meissner, 900 - Jardim Botânico

Curitiba - PR, 80.210-170 - Brasil

Recebido em: 08/04/2016 - Aprovado em: 30/05/2016 - Publicado em: 20/06/2016 DOI: 10.18677/Enciclopedia_Biosfera_2016_052

\begin{abstract}
Devido à carência de modelos simples, robustos e precisos para estimação de volume em Araucaria angustifolia, a despeito de muitos trabalhos já realizados, este estudo objetivou avaliar o emprego do fator de forma por classe diamétrica em comparação com modelos de regressão, visando a estimativa volumétrica de um povoamento de araucária em Quedas do Iguaçu - PR. Para isso, 50 árvores foram cubadas rigorosamente e três técnicas de estimação foram comparadas estatisticamente: fator de forma médio, fatores de forma médios por classes de diâmetro; e equações de regressão. Os fatores de forma individuais variaram de 0,49 a 0,97 , com média de 0,59, ao passo que os valores médios por classe variaram de 0,54 a 0,69. O modelo de Spurr logaritmizado apresentou o melhor ajuste entre os sete modelos de regressão testados. Além disso, os fatores médios por classes de diâmetro acarretaram em estimativas volumétricas mais precisas e acuradas entre as três técnicas empregadas, quebrando o paradigma de que as equações volumétricas sempre geram estimativas de melhor qualidade, quando comparadas ao fator de forma. Todavia, a aplicação do fator de forma médio geral resulta em estimativas enviesadas para menos (subestimativas), notadamente para as árvores de maior diâmetro. Com isso, concluiu-se que o uso de fatores de forma por classe de diâmetro é a melhor opção no presente caso.
\end{abstract}

PALAVRAS-CHAVE: inventário florestal, modelagem volumétrica, pinheiro-doParaná 


\title{
VOLUMETRIC ESTIMATION OF Araucaria angustifolia (Bert.) O. Kuntze THROUGH FORM FACTOR IN DIAMETRIC CLASSES AND REGRESSION MODELS
}

\begin{abstract}
Due to lack of simple, robust and accurate models for volumetric estimation in Araucaria angustifolia, despite many previous works, this study aimed to evaluate the use of the form factor by diametric class compared with regression models to estimate volume in an Araucaria stand situated in Quedas do Iguaçu - PR. A total of 50 trees had their volume determined. Three estimation techniques were compared statistically: medium form factor, medium form factors by diameter classes and regression equations. The individual form factors ranged from 0.49 to 0.97 , with an average of 0.59 . The average values per class ranged from 0.54 to 0.69 . Among the seven regression models tested the logarithmic Spurr gave the best performance. The average factors for diameter classes was the technique that provided the most precise and accurate volume estimates, breaking the paradigm that always volumetric equations generate better quality results compared to the form factor. However, the application of the general medium form factor results in biased estimates for less (underestimation), especially for the thickest trees. For this, it was concluded that the use of form factors by diameter class is the best option in this case.
\end{abstract}

KEYWORDS: forest inventory, volumetric modeling, Parana-pine

\section{INTRODUÇÃO}

O volume é, em muitos casos, a principal variável de um inventário florestal. Todavia, a sua determinação direta é dispendiosa, pois requer a cubagem rigorosa por métodos destrutivos ou procedimentos que demandam tempo e recursos. Apenas em algumas situações especiais, como em povoamentos florestais em área pequenas, a determinação direta do volume é considerada factível (SANQUETTA et al., 2014).

Assim, os métodos indiretos de estimativa do volume são necessários e utilizados nos inventários florestais, em que se medem os diâmetros à altura do peito e parte das alturas das árvores em pé. As equações de volume, ajustadas via de análise de regressão, são consideradas umas das opções mais adequadas para esse fim, pois os conhecimentos estatísticos e o emprego da computação se tornaram atualmente correntes no setor florestal. Ademais, as equações de volume são robustas e fáceis de aplicar.

Apesar do amplo uso das equações volumétricas em estimativas madeireiras, ainda é usual o emprego de uma medida denominada de fator de forma, a qual corresponde à um procedimento ainda mais simples, em que pressupõem que o volume de uma árvore pode ser calculado a partir da fórmula geométrica do cilindro reduzida pela sua conicidade, sendo expresso por um fator redutor constante. $O$ fator de forma é uma opção metodológica a ser considerada em situações em que não há equações ajustadas disponíveis e quando se deseja dar agilidade aos cálculos que compõem o inventário. Ele fornece estimativas confiáveis, desde que haja o controle em vias de tipologia florestal, espécie e classe diamétrica (FIGUEIREDO et al., 2009).

No Brasil, as espécies florestais exóticas predominam nas plantações florestais comerciais para fins de suprimento de madeira (IBÁ, 2014). Entre as 
espécies nativas mais plantadas, encontra-se a araucária (Araucaria angustifolia (Bertol.) O. Kuntze), com 11.343 hectares no país (ABRAF, 2013). Por ser uma espécie que consta na lista das ameaçadas de extinção da flora brasileira (BRASIL, 1992), o manejo de populações naturais dessa espécie atualmente é um assunto bastante controverso. Na atualidade, a principal fonte de madeira de $A$. angustifolia que disponível no mercado provém de plantações (FIGUEIREDO FILHO et al., 2015). Por isso, é fundamental investir para viabilizar o plantio comercial em larga escala, tendo em vista a sua enorme importância econômica, ambiental e cultural. Por meio de tal prática, haverá menor pressão sobre os remanescentes nativos e utilização racional desse precioso recurso madeireiro do Brasil.

Para viabilizar o manejo de plantações de araucária, é indispensável gerar conhecimentos e tecnologias aplicadas, que sejam simples, robustas e confiáveis. Além disso, a quantificação do volume via inventários florestais se insere nesse contexto. Dessa forma, o presente estudo teve como objetivo avaliar a performance do emprego do fator de forma por classe diamétrica nas estimativas de volume, ao compará-las com as obtidas por modelos volumétricos de regressão, em um povoamento de Araucaria angustifolia (Bertol.) O. Kuntze localizados na região de Quedas do Iguaçu - PR.

\section{MATERIAL E MÉTODOS}

Os dados utilizados para este estudo advêm de um povoamento comercial de Araucaria angustifolia (Bertol.) O. Kuntze, com 16 anos de idade na época da coleta. O povoamento localiza-se no município de Quedas do Iguaçu, Sudoeste do estado do Paraná. De acordo com a classificação de Köppen, o clima é do tipo subtropical úmido, mesotérmico, com verões quentes e geadas pouco frequentes, com chuvas concentradas nos meses de verão, a temperatura média do mês mais quente é superior a $22^{\circ} \mathrm{C}$ e do mês mais frio é inferior a $18^{\circ} \mathrm{C}$, não apresentando estação seca definida.

Para este estudo, 50 árvores de araucária foram cubadas baseando-se na distribuição diamétrica do povoamento (Tabela 1), cujo método de cubagem empregado foi de Hohenadl (SANQUETTA et al., 2014), com seções relativas. O volume calculado foi o total com casca, da base até o ápice da árvore.

TABELA 1. Frequência de árvores cubadas por classe diamétrica para a estimativa de volume um povoamento de Araucaria angustifolia em Quedas do Iguaçu - PR

\begin{tabular}{lll}
\hline Classe de diâmetro $(\mathrm{cm})$ & Centro de classe $(\mathrm{cm})$ & Frequência \\
\hline$\leq 7,9$ & 4,5 & 5 \\
$8,0-14,9$ & 11,5 & 7 \\
$15,0-21,9$ & 18,5 & 6 \\
$22,0-28,9$ & 25,5 & 6 \\
$29,0-35,9$ & 32,5 & 6 \\
$36,0-42,9$ & 39,5 & 6 \\
$43,0-49,9$ & 46,5 & 6 \\
$\geq 50,0$ & 53,5 & 8 \\
\hline Total & & 50 \\
\hline
\end{tabular}



testados:

Além disso, três métodos distintos de estimativas dos volumes foram

1) Aplicação do fator de forma artificial médio para as 50 árvores;

2) Aplicação do fator de forma artificial específico para cada classe diamétrica; e

3) Modelos volumétricos de regressão.

\section{Técnica 1 - Fator de forma médio}

O fator de forma artificial médio valor médio geral para todas as 50 árvores $(\bar{f})$ foi calculado com base no diâmetro a 1,30 m (dap), da seguinte forma:

$\bar{f}=\frac{\sum_{i=1}^{50} f_{i}}{50}$ (equação 1)

Em que:

$f_{i}=\frac{v_{i_{\text {real }}}}{v_{i \text { cilindro }}}=$ fator de forma de cada indivíduo (equação 2);

$v_{\text {real }}=$ volume real obtido via cubagem rigorosa $\left(\mathrm{m}^{3}\right) ; \mathrm{e}$

$v_{\text {cilindro }}=$ volume do cilindro calculado a partir do dap. estimado:

Por conseguinte, o volume de cada árvore $i$ por essa técnica foi assim

$$
\hat{v}_{i}=\frac{\pi d a p_{i}^{2}}{40.000} h_{i} \cdot \bar{f} \text { (equação 3) }
$$

\section{Técnica 2 - Fator de forma médio por classe de diâmetro}

Nesta técnica, calculou-se um fator de forma artificial médio para cada classe diamétrica, como segue:

$\bar{f}_{j}=\frac{\sum_{i=1}^{n_{j}} f_{i}}{n_{j}}$ (equação 4)

Sendo:

$f_{i}=$ fator de forma de cada indivíduo, como definido previamente; e

$n_{j}=$ número de árvores da classe diamétrica $j$. estimado:

$$
\hat{v}_{i}=\frac{\pi d a p_{i}^{2}}{40.000} h_{i} \bar{f}_{j} \text { (equação 5) }
$$

Nesse caso, o fator de forma a ser aplicado foi o valor médio da classe à qual a árvore pertenceu.

\section{Técnica 3 - Equações volumétricas}

Foram testados sete modelos matemáticos para estimativa volumétrica em função de dap e altura das árvores (Tabela 2), em que os ajustes foram efetuados pelo método de mínimos quadrados ordinários, cuja discrepância logarítmica dos modelos logarítmicos (9 a 12) foi corrigida pelo Fator de Correção de Meyer. 
TABELA 2. Modelos de regressão testados para estimar o volume total de árvores individuais de um povoamento de Araucaria angustifolia em Quedas do Iguaçu - PR

\begin{tabular}{lll}
\hline Denominação & Modelo & Equação \\
\hline Reta & $\hat{v}_{i}=\beta_{0}+\beta_{1}\left(\right.$ dap $\left._{i}\right)$ & 6 \\
Kopezky-Gehrardt & $\hat{v}_{i}=\beta_{0}+\beta_{1}\left(\right.$ dap $\left._{i}^{2}\right)$ & 7 \\
Spurr & $\hat{v}_{i}=\beta_{0}+\beta_{1}\left(\right.$ dap $\left._{i}^{2} h_{i}\right)$ & 8 \\
Husch & $\ln \left(\hat{v}_{i}\right)=\beta_{0}+\beta_{1}\left(\operatorname{In}\left(\right.\right.$ dap $\left.\left._{i}\right)\right)$ & 9 \\
Husch modificado & $\ln \left(\hat{v}_{i}\right)=\beta_{0}+\beta_{1}\left(\ln \left(\right.\right.$ dap $\left.\left._{i}^{2}\right)\right)$ & 10 \\
Schumacher-Hall & $\ln \left(\hat{v}_{i}\right)=\beta_{0}+\beta_{1}\left(\ln \left(\right.\right.$ dap $\left.\left._{i}\right)\right)+\beta_{2}\left(\operatorname{In}\left(h_{i}\right)\right)$ & 11 \\
Spurr log & $\ln \left(\hat{v}_{i}\right)=\beta_{0}+\beta_{1}\left(\ln \left(\right.\right.$ dap $\left.\left._{i}{ }^{2} h_{i}\right)\right)$ & 12 \\
\hline
\end{tabular}

Em que: In = logaritmo natural; $\hat{v}_{i}=$ volume estimado da árvore $\left(\mathrm{m}^{3}\right) ; h_{i}=$ altura total da árvore $(\mathrm{m}) ;$ dap $_{i}=$ diâmetro à altura do peito $(\mathrm{cm})$; e $\beta_{0}, \beta_{1}$ e $\beta_{2}=$ coeficientes de regressão.

A seleção do melhor modelo, entre os sete testados, foi feita com base nos seguintes critérios: coeficiente de determinação ajustado ( $R^{2}$ ajustado), erro padrão da estimativa em percentagem (Syx\%) e análise gráfica de resíduos (SANQUETTA et al., 2014). Foram, também, calculadas as estatísticas viés, precisão e acurácia para comparação entre os modelos (PRETZSCH, 2009).

\section{RESULTADOS E DISCUSSÃO}

\section{Técnica 1 - Fator de forma médio}

O fator de forma médio calculado para as 50 árvores amostradas foi de 0,59 , com variação de 0,49 a 0,97 , sendo o desvio padrão de 0,0686 e o coeficiente de variação igual a $11,70 \%$.

\section{Técnica 2 - Fator de forma médio por classe de diâmetro}

Os fatores de forma médios por classe variaram de 0,54 a 0,69 (Tabela 3). Com isso, percebe-se uma tendência de decréscimo do fator de forma no sentido das maiores classes diamétricas, o que demonstra que árvores de diâmetros inferiores possuem menor conicidade em relação às de maior porte.

TABELA 3. Fatores de forma médios por classe de diâmetro em árvores cubadas de um povoamento de Araucaria angustifolia em Quedas do Iguaçu - PR

\begin{tabular}{lll}
\hline Classe de diâmetro $(\mathrm{cm})$ & Centro de classe $(\mathrm{cm})$ & Fator de forma \\
\hline$\leq 7,9$ & 4,5 & 0,69 \\
$8,0-14,9$ & 11,5 & 0,61 \\
$15,0-21,9$ & 18,5 & 0,58 \\
$22,0-28,9$ & 25,5 & 0,57 \\
$29,0-35,9$ & 32,5 & 0,59 \\
$36,0-42,9$ & 39,5 & 0,60 \\
$43,0-49,9$ & 46,5 & 0,55 \\
$\geq 50,0$ & 53,5 & 0,54 \\
\hline
\end{tabular}




\section{Técnica 3 - Equações volumétricas}

De modo geral, os modelos testados apresentaram altos valores de coeficiente de determinação, acima de 0,9 (Tabela 4), ao passo que os erros padrão da estimativa em percentagem foram mais baixos para os modelos 3,6 e 7 , sendo inferiores a 10\%, o que acarretou em uma característica desejável para estimativas volumétricas. Os demais apresentaram valores de Syx\% superiores, indicando que, apesar do alto valor de $R^{2} a j$, seu ajuste não foi satisfatório.

TABELA 4. Estatísticas de ajuste e de precisão dos modelos de regressão utilizados para estimar o volume de árvores individuais de um povoamento de Araucaria angustifolia em Quedas do Iguaçu - PR

\begin{tabular}{llllll}
\hline Modelo & $\beta_{0}$ & $\beta_{1}$ & $\beta_{2}$ & $R^{2}$ ajustado & Sxy \% \\
\hline 1 & $-0,693360$ & 0,060489 & - & 0,9227 & 28,76 \\
2 & $-0,052980$ & 0,001018 & - & 0,9712 & 20,46 \\
3 & 0,043780 & 0,000042 & - & 0,9999 & 8,82 \\
4 & $-8,487540$ & 2,422325 & - & 0,9515 & 24,18 \\
5 & $-8,487540$ & 1,211163 & - & 0,9515 & 24,18 \\
6 & $-9,504490$ & 2,001555 & 0,827340 & 0,9999 & 9,70 \\
7 & $-9,668700$ & 0,964990 & & 0,9999 & 8,33 \\
\hline
\end{tabular}

Há uma tendência de aceitar, como ajustes satisfatórios, as equações que resultem em valores altos de $R^{2}$, entretanto, como verificado no presente estudo, essa recomendação não acarreta em verdade absoluta, sendo um fato comprovado pelos valores altos de erro padrão (Tabela 4) e pela inadequada dispersão dos resíduos (Figura 1). Ademais, pelos gráficos de distribuição dos resíduos, ficou evidente que 0 modelo 1 foi totalmente inadequado, resultando em estimativas negativas para as pequenas árvores, enquanto os modelos 2, 4 e 5 apresentaram melhor desempenho, mas geraram estimativas tendenciosas para os maiores indivíduos, o que é comprometedor, tendo em vista que são essas as árvores que mais produzem em termos volumétricos.

Os modelos 2, 6 e 7 produziram estimativas precisas e sem tendência, com altos valores de coeficiente de determinação, baixos erros padrão da estimativa e distribuição equilibrada dos resíduos ao longo da linha de ajuste. Portanto, eles podem ser considerados satisfatórios. Entre eles, o que proporcionou as melhores estatísticas de ajuste foi o 7, denominado Spurr logaritimizado.

Segundo OLIVEIRA et al. (2011), ao testaram sete modelos matemáticos para estimar o volume de araucária em Rio Negro - PR, concluíram que o modelo de Schumacher-Hall foi o mais satisfatório entre os avaliados. Apesar de a idade do povoamento no momento da avaliação dos referidos autores ser de 44 anos, sendo superior ao deste estudo, e da amplitude diamétrica ser inferior àquela encontrada neste trabalho, os níveis de ajuste do trabalho previamente citado são próximos dos encontrados nesta pesquisa. A espécie em questão tem a forma muito definida e as correlações de dap e altura com o volume são geralmente muito fortes $(0,95$ do volume com o dap e de 0,84 com a altura, neste estudo). Esse fato favorece a obtenção de estimativas volumétricas de qualidade para a espécie. Porém, nem sempre bons indicadores estatísticos isolados, notadamente o $R^{2}$, pode significar estimativas precisas e sem tendência.

\section{Síntese comparativa}

Comparando as três técnicas de estimação do volume (Tabela 5), observouse que a técnica 1 (fator de forma médio) gerou um viés negativo de 
aproximadamente $5 \%$, ou seja, uma subestimativa dessa magnitude. A técnica 2 (fator de forma médio por classe) produziu viés muito baixo, o mesmo aconteceu com a técnica 3 (equações volumétricas), com exceção dos modelos 4 e 5.

TABELA 5. Viés, precisão e acurácia das técnicas avaliadas para estimar o volume de árvores individuais de um povoamento de Araucaria angustifolia em Quedas do Iguaçu - PR

\begin{tabular}{lllll}
\hline Técnica & Modelo & Viés\% & Precisão\% & Acurácia\% \\
\hline Fator de forma médio & - & $-4,98$ & 12,16 & 13,16 \\
Fator de forma médio por classe de diâmetro & - & $-0,19$ & 7,19 & 7,19 \\
& 1 & 0,00 & 28,46 & 28,46 \\
& 2 & 0,00 & 20,25 & 20,25 \\
Equações volumétricas & 3 & 0,00 & 8,73 & 8,73 \\
& 4 & 2,81 & 24,43 & 23,93 \\
& 5 & 2,81 & 24,43 & 23,93 \\
& 6 & $-0,10$ & 9,50 & 9,50 \\
\hline
\end{tabular}

No que tange à precisão e acurácia, a técnica 1 gerou, aparentemente, estimativas razoavelmente precisas e acuradas, inclusive superiores aos modelos volumétricos 1, 2, 4 e 5 . Por outro lado, as equações volumétricas 3,6 e 7 proporcionaram melhores valores de precisão e acurácia, inferiores a $10 \%$.

Nesse sentido, CAMPOS \& LEITE (2009) discorreram sobre o emprego do fator de forma para estimativa de volume em árvores, em que, segundo os autores, é usual utilizar um fator de forma médio, porém são várias as ressalvas para seu uso. Adicionalmente, SOUZA \& JESUS (1991) compararam equações de volume com fator de forma para espécies da Mata Atlântica no Espírito Santo, concluindo que não houve diferença significativa entre ambos; e MIGUEL et al. (2010) comentaram que o fator de forma pode ser utilizado para uma estimativa rápida do volume individual ou do povoamento, quando os plantios apresentam características semelhantes.

MIRANDA et al. (2015) compararam equações volumétricas com o fator de forma em estimativas para Eucalyptus urograndis na região Noroeste do estado de Mato Grosso. Segundo os autores, não houve diferenças estatísticas na estimativa dos volumes total e comercial com casca, utilizando o fator de forma e a melhor equação ajustada por regressão. Acrescentaram, ainda, que o fator de forma é um método muito adotado para obter estimativas rápidas do volume, mas deve ter ponderações e cuidados quanto ao seu uso, principalmente quando não for específico.

Enfim, existem vários estudos sobre o tema, alguns demonstrando que não há um ganho generalizado no emprego de equações volumétricas. Não existem estudos mais particularizados sobre empregar fatores de forma por classe de diâmetro e seus benefícios no cálculo de volumes individuais.

Neste estudo, o fato de a técnica 2 resultar nos melhores indicadores de precisão e acurácia foi uma surpresa, em relação às equações volumétricas. Observou-se que, além do baixíssimo viés, a precisão e a acurácia das estimativas com essas técnicas foram as mais satisfatórias. A qualidade das estimativas com essa técnica foi corroborada pela distribuição de resíduos (Figura 1), que demonstraram a superioridade da técnica para estimar o volume, sendo simples, não enviesada, precisa e acurada. 
KOHLER (2013) mencionou que a literatura recomenda utilizar fatores de forma variáveis por idade e por classe de diâmetro para estimar os volumes, tendo em vista as variações na forma com tais variáveis. Tal sugestão vem ao encontro deste estudo, pois verificou-se que há uma diferença numérica importante no fator de forma entre as classes de diâmetro. No caso em apreço, não há variação de idade do povoamento, contudo, caso houvesse, possivelmente seria necessário também considerá-la na estimativa volumétrica. Com isso, estudos nesse sentido merecem ser aprofundados no futuro.
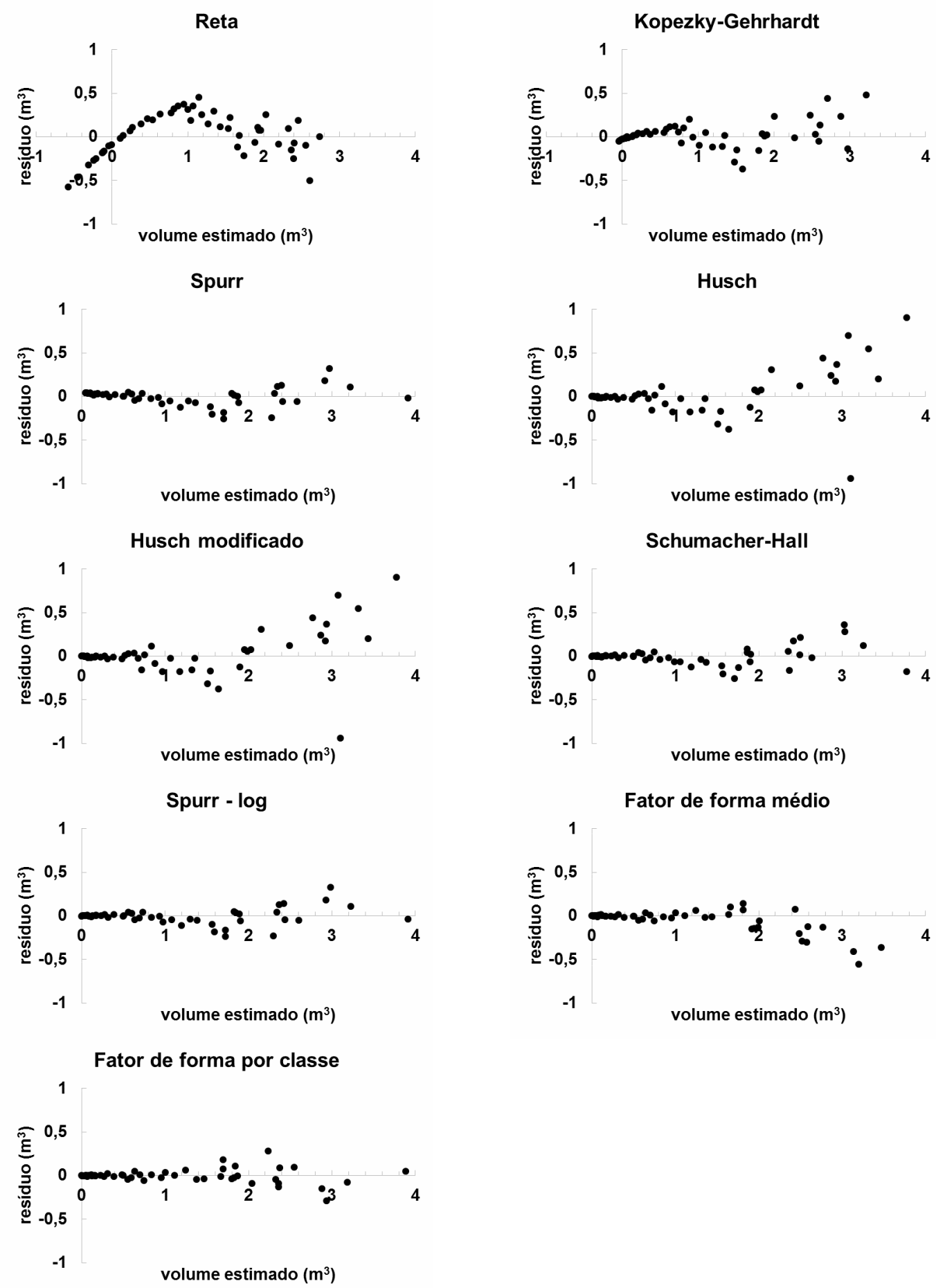

FIGURA 1. Gráficos de dispersão dos resíduos para modelos volumétricos ajustados para estimar o volume de árvores individuais de um povoamento de Araucaria angustifolia em Quedas do Iguaçu - PR 


\section{CONCLUSÕES}

- A aplicação de um fator de forma médio para estimativa de volume gera valores enviesados, principalmente para as maiores árvores;

- As equações volumétricas ajustadas com altos valores de coeficiente de determinação não necessariamente são precisas e acuradas;

- As estimativas volumétricas via equações volumétricas não são frequentemente superiores às do fator de forma;

- A aplicação de fatores de forma por classes diamétricas é a melhor opção para estimar o volume no presente estudo. Essa técnica é simples e robusta, sendo passível de ser empregada com segurança, desde que não haja variações significativas dos valores do fator de forma dentro da classe diamétrica. No presente estudo, essa técnica gera estimativas sem viés, precisas e acuradas.

\section{REFERÊNCIAS}

ABRAF - ASSOCIAÇÃO BRASILEIRA DE PRODUTORES DE FLORESTAS PLANTADAS. Anuário estatístico da ABRAF 2013 ano base 2012. Brasília, DF, 2013. $146 \mathrm{p}$.

BRASIL. Portaria no. 06-N, de 15 de janeiro de 1992. Lista oficial de espécies da flora brasileira ameaçadas de extinção. Diário Oficial (da República Federativa do Brasil), Brasília, 23 jan. 1992. p.870-872. Disponível em: <http://www.mma.gov.br/estruturas/179/_arquivos/179_05122008033646.pdf>

CAMPOS, J. C. C.; LEITE, H. G. Mensuração florestal: perguntas e respostas. 3. Ed. Viçosa: Editora UFV, 2009. 548 p.

FIGUEIREDO, E. O.; SCHROEDER, R.; PAPA, D. A. Fatores de forma para 20 espécies florestais comerciais da Amazônia. Rio Branco, Empresa Brasileira de Pesquisa Agropecuária (EMBRAPA). Comunicado técnico 173, 2009. 4p. Disponível em <https://www.infoteca.cnptia.embrapa.br/bitstream/doc/697548/1/cot173.pdf>.

FIGUEIRDO FILHO, A.; RETSLAFF, F. A. S.; KOHLER, S. V.; BECKER, M.; BRANDES, D. Efeito da Idade no Afilamento e Sortimento em Povoamentos de Araucaria angustifolia. Floresta e Ambiente, v. 22, n. 1, p. 50-59, 2015. Disponível em: < http://dx.doi.org/10.1590/2179-8087.080114>. doi: 10.1590/21798087.080114 .

IBÁ - INDÚSTRIA BRASILEIRA DE ÁRVORES. Anuário estatístico da indústria brasileira de árvores: ano base 2013. 2014. 97 p. Disponível em: http//:www.iba.org.br. Acesso em 20/04/2015.

KOHLER, S. V. Evolução do afilamento do tronco e do sortimento em plantios de Pinus taeda nos estados do Paraná e Santa Catarina. Dissertação (mestrado). 2013. 83 f. Dissertação (Mestrado) - Pós-Graduação em Engenharia Florestal, Universidade Federal do Paraná, Curitiba, 2013.

MIGUEL, E. P.; CANZI, L. F.; RUFINO, R. F.; SANTOS, G. A. Ajuste de modelo volumétrico e desenvolvimento de fator de forma para plantios de Eucalyptus grandis 
localizados no município de Rio Verde - GO. Enciclopédia Biosfera, v. 6, n. 1, p. 1 13, 2010.

MIRANDA, D. L. C.; JUNIOR, V. B.; GOUVEIA, D. M. Fator de forma e equações de volume para estimativa volumétrica de árvores em plantio de Eucalyptus urograndis, Scientia Plena, v. 11, n. 2, p.1-8, 2015.

OLIVEIRA, M. F.; MATTOS, P. P.; BRAZ, E. M.; ROSOT, M. A. D.; ROSOT, N. C.; SANTOS, W. C. Equação de volume para um plantio de Araucaria angustifolia em Rio Negro - PR. Colombo, Empresa Brasileira de Pesquisa Agropecuária (EMBRAPA). Comunicado técnico 271. 2011. Disponível em < https://www.infoteca.cnptia.embrapa.br/infoteca/bitstream/doc/898047/1/CT275.pdf>

PRETZSCH, H. Forest dynamics, growth and yield: from measurement to model. Berlin: Springer-Verlag, 2009. 664p.

SANQUETTA, C. R.; CORTE, A. P. D.; RODRIGUES, A. L.; WATZLAWICK, L. F. Inventários Florestais: Planejamento e Execução. Curitiba: Multi-Graphic, 2014. $406 \mathrm{p}$.

SOUZA A. L.; JESUS, R. M. Equações de volume comercial e fator de forma para espécies da Mata Atlântica ocorrentes na Reserva Florestal da Companhia Vale do Rio Doce, Linhares, ES. Árvore, v. 15, n. 3, p. 257-273,1991. 